\title{
Modest Changes in Cerebral Glucose Metabolism in Patients with Sleep Apnea Syndrome after Continuous Positive Airway Pressure Treatment
}

\author{
Gawon Ju ${ }^{a}$ In-Young Yoon ${ }^{b}$ Sang Don Lee ${ }^{b}$ Yu Kyeong Kim ${ }^{c}$ Eunjin Yoon ${ }^{c}$ \\ Jeong-Whun Kim ${ }^{\text {d }}$ \\ a Department of Neuropsychiatry, Chungbuk National University Hospital, Cheongju, and Departments of

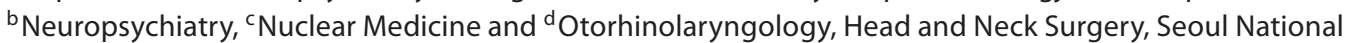 \\ University Bundang Hospital, Seongnam, Korea
}

\section{Key Words}

Sleep apnea syndrome $\cdot$ Positron emission tomography •

Continuous positive airway pressure

\begin{abstract}
Background: Decreased cerebral glucose metabolism has been reported in patients with sleep apnea syndrome (SAS), but it has yet to be decided whether cerebral glucose metabolism in SAS can be altered by continuous positive airway pressure (CPAP) treatment. Objective: The aim of this study was to evaluate cerebral glucose metabolism changes in patients with SAS after CPAP treatment. Methods: Thirteen middle-aged male patients with severe SAS [mean age 49.3 \pm 7.2 years, mean apnea-hypopnea index (AHI) $60.4 \pm 21.2]$ and 13 male controls (mean age $46.0 \pm 9.4$ years, mean AHI $4.1 \pm 3.7$ ) participated in the study. All 26 study subjects underwent fluorodeoxyglucose-positron emission tomography (FDG-PET), but SAS patients underwent FDG-PET twice, namely before and 3 months after acceptable CPAP usage. Results: Significant hypometabolism was observed in the bilateral prefrontal areas, left cuneus and left cingulate cortex of SAS patients before CPAP, and after CPAP, significant increases in cortical glucose metabolism were observed in the bilateral precentral gyri and left anterior cingulate cor-
\end{abstract}

tex. However, these improvements in hypometabolism in both areas were insufficient to reach control levels, and hypometabolism in other regions persisted after CPAP treatment. Conclusions: Reduced cerebral glucose metabolism in the precentral gyrus and the cingulate cortex in patients with SAS was modestly improved by acceptable CPAP treatment. The findings of this study suggest that acceptable CPAP usage cannot completely reverse reduced cerebral glucose metabolism in SAS patients. Further studies are required to evaluate the long-term effects of CPAP treatment with total compliance.

Copyright $\odot 2012$ S. Karger AG, Basel

\section{Introduction}

Sleep apnea syndrome (SAS) is characterized by nocturnal hypoxemia and frequent arousals during sleep caused by repetitive respiratory pauses. The estimated prevalence of SAS among middle-aged adults is $9 \%$ for women and $24 \%$ for men [1]. Furthermore, SAS is associated with significant medical, cognitive and psychological sequelae, including excessive daytime sleepiness, an increased risk of cardiovascular disease and neurocognitive consequences [2-5]. Also, SAS-related intermittent

\section{KARGER}

Fax +4161306 1234

E-Mail karger@karger.ch

www.karger.com
(C) 2012 S. Karger AG, Basel

$0025-7931 / 12 / 0843-0212 \$ 38.00 / 0$

Accessible online at:

www.karger.com/res
In-Young Yoon

Department of Neuropsychiatry

Seoul National University Bundang Hospital

300 Gumi-dong, Bundang-gu, Seongnam-si, Gyeonggi-do 463-707 (South Korea)

Tel. +82 31787 7433, E-Mail iyoon@ @nu.ac.kr 
hypoxia and sleep fragmentation might result in structural and functional changes in the brain.

Investigations that have applied neuroimaging methodologies have made important contributions to our understanding of brain structure and function in individuals with SAS. Recent evidence suggests that SAS may lead to structural brain abnormalities [6,7], and studies using functional neuroimaging techniques observe subtle cerebral changes associated with SAS. With regard to ${ }^{18} \mathrm{~F}$ fluoro-2-deoxy-D-glucose-positron emission tomography (FDG-PET) studies, two preliminary, small-sample reports have been published $[8,9]$. Recently, Yaouhi et al. [10] reported that SAS with little cognitive impairment showed right-lateralized hypometabolism in the precuneus, middle and posterior cingulated gyri and the parieto-occipital cortex.

Continuous positive airway pressure (CPAP) is the first-line treatment in SAS and is effective at improving daytime sleepiness [11] and reducing the risks of cardiovascular complications [12]. However, the superiority of CPAP over placebo with respect to cognitive functioning has not been demonstrated in placebo-controlled studies, as impairments in executive function tend to persist despite CPAP, probably due to irreversible hypoxemic damage $[13,14]$. Tonon et al. [13] also showed in a study using magnetic resonance spectroscopy that decreased cortical $\mathrm{N}$-acetylaspartate levels in SAS persisted after CPAP therapy. These studies show that some symptoms and complications of SAS are improved by CPAP, but that other abnormalities persist despite treatment.

However, it has not yet been shown whether CPAP treatment alters cerebral glucose metabolism in SAS patients. Thus, in this study, we undertook to evaluate brain glucose metabolism abnormalities in SAS patients using FDG-PET and to determine whether CPAP treatment alters glucose metabolism.

\section{Methods}

\section{Subjects}

Thirteen middle-aged, never-treated male SAS patients (mean age $49.3 \pm 7.2$ years) and 13 male controls (mean age $46.0 \pm 9.4$ years) participated in this study. All study subjects visited a sleep clinic for either snoring or suspected sleep apnea and were referred to the sleep laboratory in Seoul National University Bundang Hospital. No subject had a history of cerebrovascular or ischemic heart disease or central nervous system disorders. The 13 patients had severe SAS [apnea-hypopnea index (AHI) >30], whereas the healthy controls had an AHI of $<5$.

At baseline, patients and controls were evaluated for brain glucose metabolism by FDG-PET and completed questionnaires, in- cluding the Epworth Sleepiness Scale for daytime sleepiness, the Beck Depression Inventory for depressive mood and the Pittsburgh Sleep Quality Index for subjective sleep disturbances. After CPAP treatment for 3 months, patients were assessed again by FDG-PET and using the above-mentioned questionnaires. The study protocol was approved by the Institutional Review Board of the Seoul National University Bundang Hospital, and all subjects provided written informed consent.

\section{Polysomnography}

Overnight polysomnography (PSG) was performed using an Embla $^{\mathrm{TM}}$ N 7000 recording system (Embla, Reykjavik, Iceland) and standard electrodes and sensors. Every 30-second epoch of the PSG was scored based on the criteria of Rechtschaffen and Kales [15]. Apnea was defined as the complete cessation of airflow for at least $10 \mathrm{~s}$. Hypopnea was defined as a substantial (>50\%) reduction in airflow for at least $10 \mathrm{~s}$ or a moderate reduction in airflow for at least $10 \mathrm{~s}$ associated with EEG arousal or oxygen desaturation $(\geq 4 \%)$ [16]. AHI was defined as the total number of apnea and hypopnea episodes per hour of sleep, and the oxygen desaturation index was defined as the number of oxygen desaturations $(\geq 4 \%)$ per hour of sleep.

\section{Positive Airway Pressure Treatment}

After the baseline polysomnographic evaluation, a secondnight PSG was performed for the titration of CPAP. The optimal pressure was the minimum pressure able to eliminate apneas and hypopneas in each patient [17]. After CPAP treatment for at least 3 months, data regarding CPAP usage and mean AHI during CPAP were obtained from data cards inside the CPAP machine. CPAP compliance was defined as days of CPAP use (for at least $4 \mathrm{~h} /$ day) expressed as a percentage of total study period days. Subjects with a CPAP compliance of $70 \%$ were defined as having acceptable compliance [18].

\section{Image Acquisition}

All patients underwent MRI and FDG-PET scan in the morning on the same days. A high-resolution $\mathrm{T}_{1}$-weighted volume MRI scan was conducted to exclude subjects with any cerebrovascular problem. A Philips Intera 1.5T MRI system was used to acquire high-resolution T1 anatomical brain images. Anatomical brain images were acquired using a 3 -dimensional (3D) $\mathrm{T}_{1}$-weighted gradient echo (T1TFE) sequence using the following parameters: $\mathrm{TR}=8.0381 \mathrm{~ms}, \mathrm{TE}=3.6828 \mathrm{~ms}$, flip angle $=8^{\circ}, 175$ slices, thickness $=1 \mathrm{~mm}$, matrix size $=256 \times 256$.

FDG-PET images were acquired using a Philips Allegro PET scanner operating in 3D mode. All subjects fasted for at least $6 \mathrm{~h}$ before scanning. After being given an intravenous injection of 4.8 $\mathrm{MBq} / \mathrm{kg}$ FDG in a dimly lit, quiet waiting room, each study subject was instructed to remain lying comfortably for $40 \mathrm{~min}$ for FDG equilibration. A 10-min emission scan and an attenuation map using a Cs-137 transmission source were then obtained. Attenuation-corrected images were reconstructed using the $3 \mathrm{D}$ rowaction maximum likelihood algorithm and a $3 \mathrm{D}$ image filter of $128 \times 128 \times 90$ matrices with a pixel size of $2 \times 2 \times 2 \mathrm{~mm}$.

FDG-PET Analysis

Preprocessing and statistical analysis were performed using SPM5 (Statistical Parametric Mapping, Wellcome Department of Imaging Neuroscience, London, UK, http://www.fil.ion.ucl.ac. 
uk/spm) implemented in Matlab 7.6 (The Mathworks, Mass., USA). FDG-PET images were normalized against a standard PET template, developed and distributed by the Montreal Neurological Institute, and then smoothed with a $12-\mathrm{mm}$ full-width-at-halfmaximum isotropic Gaussian kernel. Brain glucose metabolism at each voxel was proportionally scaled according to the mean FDG uptake value of the pons. The paired-sample t test was used to determine significant differences in brain regional metabolism after CPAP treatment. The difference was considered significant when a cluster consisting of at least 50 contiguous voxels exceeded a threshold height of $\mathrm{p}<0.005$ (uncorrected for multiple comparison).

\section{Statistical Analysis}

SPSS version $17 \mathrm{~K}$ for Windows (SPSS Inc., Chicago, Ill., USA) was used for the statistical analysis. Results are presented as means \pm standard deviation. Differences between groups in terms of parametric clinical variables were assessed by using the two-sample paired t test or the independent $t$ test. Furthermore, mean uptake in the brain regions which showed significant changes after CPAP on SPM analyses was extracted from pre- and posttreatment scans using MarsBaR (http://marsbar.sourceforge. net). Pearson's or Spearman's correlation coefficients were calculated to determine relationships between glucose metabolism changes and clinical or polysomnographic variables.

\section{Results}

Table 1 shows the demographic and clinical characteristics of the study subjects. The mean age of the 13 SAS patients was $49.8 \pm 7.0$ years, and their mean AHI was $60.3 \pm 21.2$. The mean duration of CPAP treatment was $93.8 \pm 19.8$ days (range 90-119 days), and mean CPAP compliance was $78.9 \pm 14.5 \%$. Of the 13 SAS patients, 11 patients had acceptable compliance and the other $2 \mathrm{had}$ compliance of 58.8 and $47 \%$. Body mass index, oxygen desaturation index and education levels are also presented in table 1. During CPAP treatment, the mean AHI of the patients was significantly decreased from $60.3 \pm 21.2$ at baseline to $3.8 \pm 2.2(\mathrm{p}<0.05)$ after CPAP. Daytime sleepiness and quality of sleep were significantly improved after CPAP $(\mathrm{p}<0.05)$. Controls and patients were similar for all demographic measures except body mass index.

SAS patients showed hypometabolism in the bilateral lateral prefrontal areas, bilateral precentral gyri, left cuneus, right angular gyrus, left superior parietal lobule and left cingulate cortex versus controls. After CPAP treatment, cerebral glucose metabolism increased in the bilateral precentral gyri and left anterior cingulate cortices (ACC; all p < 0.005; fig. 1). However, these improvements observed after CPAP treatment fell short of control levels (table 2). In addition, hypometabolism persisted af-
Table 1. Demographic and clinical characteristics of the study subjects

\begin{tabular}{lccl}
\hline & $\begin{array}{l}\text { Controls } \\
(\mathrm{n}=13)\end{array}$ & $\begin{array}{l}\text { SAS } \\
\text { pre-CPAP } \\
(\mathrm{n}=13)\end{array}$ & $\begin{array}{l}\text { SAS } \\
\text { post-CPAP } \\
(\mathrm{n}=13)\end{array}$ \\
\hline Age, years & & & \\
BMI & $46.0 \pm 9.4$ & $49.8 \pm 7.0$ & \\
PSQI & $23.7 \pm 1.9^{\mathrm{a}}$ & $30.6 \pm 4.6$ & $30.1 \pm 4.4$ \\
ESS & $5.8 \pm 2.1$ & $6.4 \pm 3.1^{\mathrm{b}}$ & $3.7 \pm 1.8$ \\
BDI & $9.8 \pm 5.4$ & $12.3 \pm 5.7^{\mathrm{b}}$ & $6.7 \pm 5.3$ \\
AHI, n/h & $3.2 \pm 3.6$ & $6.7 \pm 5.5$ & $3.7 \pm 2.8$ \\
ODI, n/h & $4.1 \pm 3.7^{\mathrm{a}}$ & $60.3 \pm 21.2^{\mathrm{b}}$ & $3.8 \pm 2.2$ \\
Time of SpO $<90 \%, \%$ & $1.6 \pm 1.3^{\mathrm{a}}$ & $54.6 \pm 23.0$ & - \\
Total sleep time, min & $386.3 \pm 3.01 .1$ & $401.8 \pm 47.2$ & - \\
Sleep efficiency, \% & $84.2 \pm 10.5$ & $87.6 \pm 7.7$ & - \\
Stage 1, \% of SPT & $9.1 \pm 3.1^{\mathrm{a}}$ & $28.0 \pm 8.5$ & - \\
Stage 2, \% of SPT & $52.5 \pm 8.7^{\mathrm{a}}$ & $42.9 \pm 7.5$ & - \\
Stage 3 + 4, \% of SPT & $5.8 \pm 5.8$ & $2.0 \pm 4.2$ & - \\
Stage R, \% of SPT & $18.8 \pm 4.9$ & $17.6 \pm 5.4$ & - \\
WASO, min & $65.1 \pm 52.3$ & $42.3 \pm 24.7$ & - \\
Respiratory arousal index & $3.0 \pm 2.4^{\mathrm{a}}$ & $51.7 \pm 22.5$ & - \\
CPAP pressure, mm $\mathrm{H}_{2} \mathrm{O}$ & - & $11.2 \pm 2.1(9-15)$ \\
CPAP duration, days & - & $93.8 \pm 19.8(90-119)$ \\
CPAP compliance, \% & - & $78.9 \pm 14.5(47-97.9)$ \\
\hline
\end{tabular}

Values in parentheses represent ranges. ${ }^{a} \mathrm{p}<0.05$ : control versus SAS pre-CPAP; ${ }^{b} \mathrm{p}<0.05$ : SAS pre-CPAP versus SAS postCPAP. BMI = Body mass index; PSQI = Pittsburgh Sleep Quality Index; ESS = Epworth Sleepiness Scale; BDI = Beck Depression Inventory; ODI = oxygen desaturation index; SPT = sleep period time; WASO = wake after sleep onset.

ter CPAP treatment in other regions that showed reduced metabolism before CPAP (table 2). Correlational analysis between changes in clinical variables such as AHI, Epworth Sleepiness Scale and Pittsburgh Sleep Quality Index and changes in glucose metabolism in the bilateral precentral gyri and left ACC after CPAP treatment failed to reveal any relation.

\section{Discussion}

In the present study, significant hypometabolism was found in the bilateral prefrontal areas, precuneus and left ACC of SAS patients, which are known to be predominantly affected by hypoxia $[19,20]$. This finding is largely consistent with previous PET studies, in which SAS patients with little cognitive dysfunction showed rightlateralized hypometabolism in the precuneus, middle and posterior cingulated gyri and the parieto-occipital 


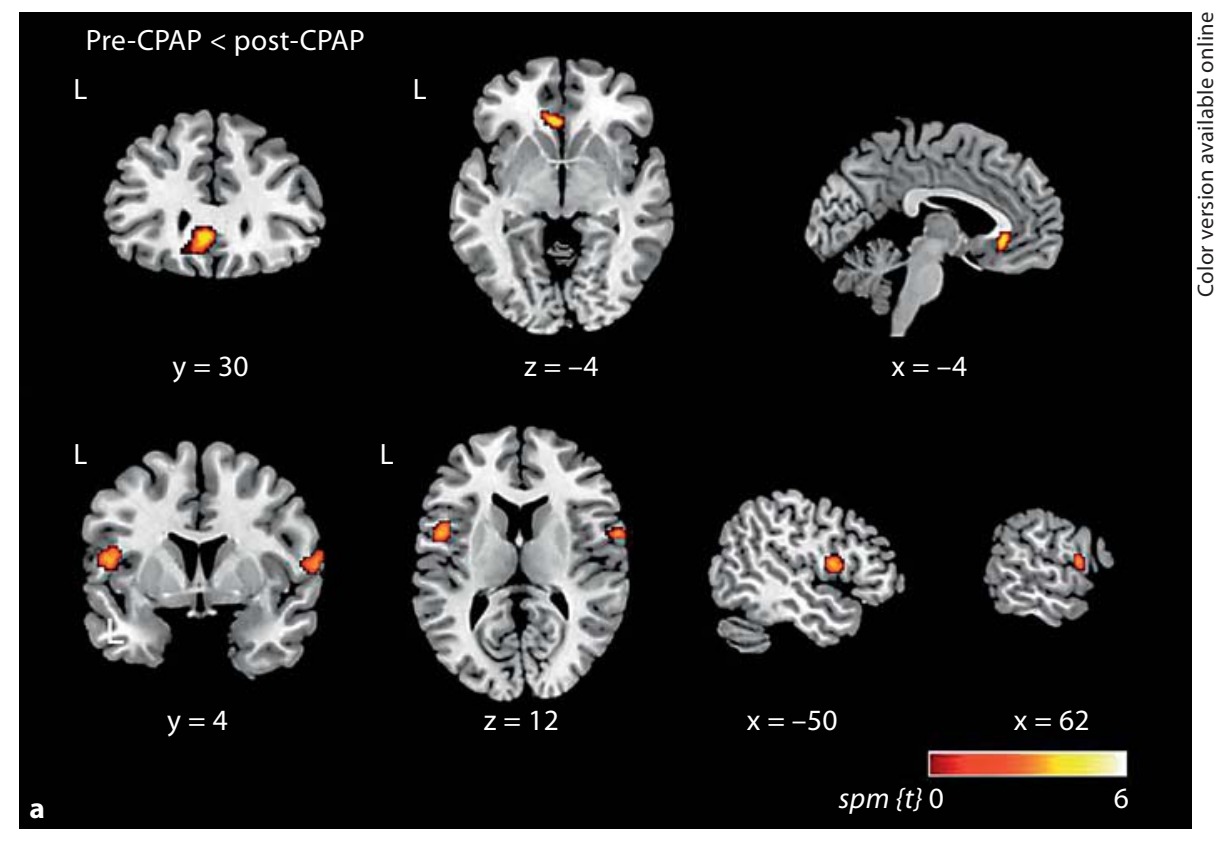

Fig. 1. Changes in cerebral glucose metabolism after 3 months of CPAP treatment. a A significant increase in cerebral metabolism after CPAP treatment was observed in the left ACC and bilateral precentral gyri (all $\mathrm{p}<0.005$; cluster size $>50$ ). $\mathrm{L}=$ Left. b Data points represent relative metabolism in these three regions of individual subjects before and after treatment. The $y$-axis denotes relative cerebral glucose metabolism, which was normalized against FDG uptake by the whole brain.

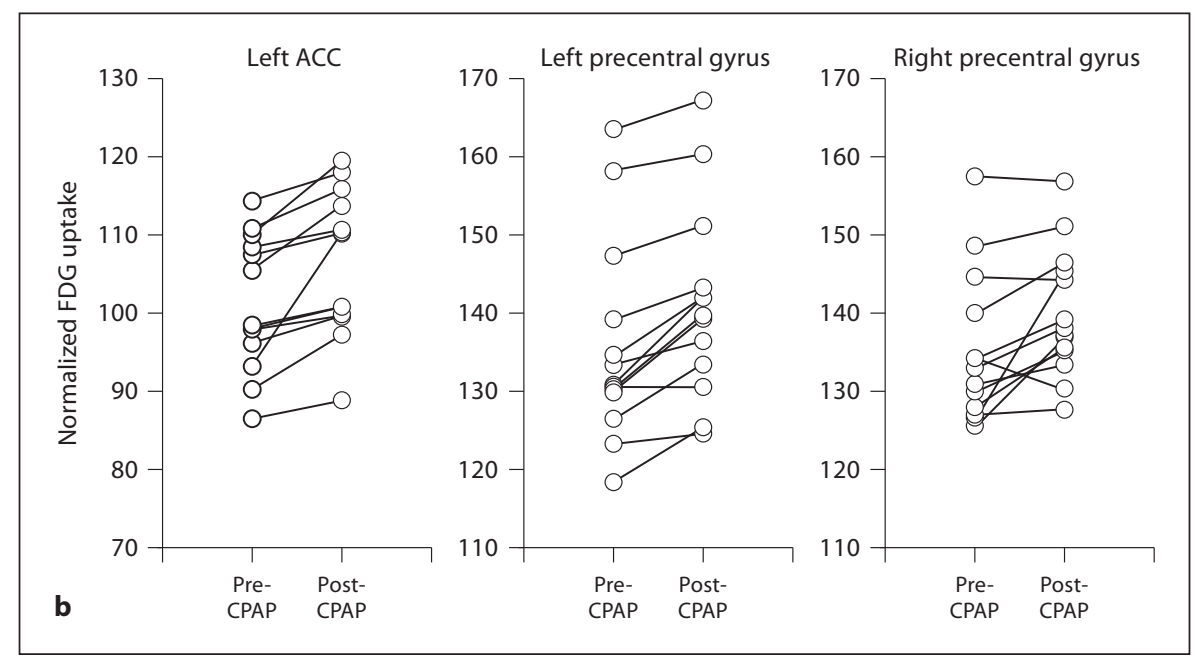

cortex [8-10]. These metabolic changes may precede future cognitive impairments in SAS, even if there are only minor cognitive deficits at the time of measurement [10, 13]. With regard to causes of the hypometabolism, apneaassociated microarousals may be correlated with the hypometabolism in SAS [10], but several experimental models of intermittent hypoxia [21,22], as well as findings in SAS [13] and chronic obstructive pulmonary disease patients $[23,24]$, provide evidence that hypoxia can be the main cause of hypometabolism in SAS.

In the present study, it was found that cortical glucose metabolism in the bilateral precentral gyri and left ACC were modestly increased after acceptable CPAP treat- ment. This finding of a modest improvement in glucose metabolism after CPAP treatment partly concurs with the findings of Canessa et al. [25], who observed that cognitive impairments and gray matter volume deficits in SAS were recovered by CPAP. They reported significant improvements in memory, attention and executive function that paralleled gray matter volume increases in hippocampal and frontal structures. In addition, Castronovo et al. [20] suggested that an overrecruitment of brain regions by SAS patients was reduced by CPAP treatment in the ACC and hippocampus. These findings suggest that CPAP treatment induces neural compensation and reduces cerebral dysfunction. 
Table 2. Comparison of glucose metabolism between normal controls and severe SAS patients after CPAP treatment

\begin{tabular}{|c|c|c|c|c|c|c|}
\hline \multirow[t]{2}{*}{ Region } & \multicolumn{3}{|c|}{ MNI coordinates } & \multirow{2}{*}{$\begin{array}{l}\text { Brodmann } \\
\text { area }\end{array}$} & \multirow[t]{2}{*}{ t score } & \multirow[t]{2}{*}{ z score } \\
\hline & $\mathrm{x}$ & $\mathrm{y}$ & $\mathrm{z}$ & & & \\
\hline L precentral gyrus & -66 & -2 & 20 & 6 & 4.90 & 4.04 \\
\hline $\mathrm{R}$ precentral gyrus & 58 & -2 & 52 & 6 & 4.16 & 3.57 \\
\hline $\mathrm{L}$ middle frontal gyrus & -34 & 28 & 54 & $8 / 6 / 10$ & 4.72 & 3.93 \\
\hline $\mathrm{R}$ middle frontal gyrus & 40 & 62 & -2 & 10 & 4.15 & 3.56 \\
\hline $\mathrm{R}$ inferior frontal gyrus & 62 & 24 & 22 & $45 / 46 / 9$ & 4.22 & 3.61 \\
\hline $\mathrm{L}$ inferior frontal gyrus & -52 & 40 & -8 & 47 & 3.72 & 3.27 \\
\hline $\mathrm{R}$ superior frontal gyrus & 30 & 42 & 48 & $8 / 9$ & 3.93 & 3.42 \\
\hline L superior frontal gyrus & -16 & 32 & 60 & $8 / 10 / 9$ & 3.90 & 3.40 \\
\hline $\mathrm{R}$ angular gyrus & 54 & -72 & 40 & 39 & 3.92 & 3.41 \\
\hline L cuneus & -10 & -106 & -2 & 18 & 3.88 & 3.39 \\
\hline L superior parietal lobule & -30 & -82 & 48 & 7 & 3.49 & 3.11 \\
\hline L cingulate cortex & -2 & -16 & 34 & 23 & 3.01 & 2.75 \\
\hline
\end{tabular}

Impaired glucose metabolism in patients with severe SAS persisted after CPAP treatment $(\mathrm{p}<0.005, \mathrm{k}=50)$. MNI = Montreal Neurological Institute; $\mathrm{L}=$ left; $\mathrm{R}=$ right.

Frontal lobe plasticity might explain the improved glucose metabolism observed in the present study. In human and animal studies, frontal regions are known to be vulnerable to hypoxia, which generates cellular and biochemical stresses, resulting in the disruption of functional homeostasis and reductions in neuronal and glial viability [26]. Brain structural plasticity (especially in the frontal area and hippocampus) caused by environmental enrichment has been demonstrated in animal models [27], and there is evidence that cognitive training can cause plasticity in the frontal area [28]. Interestingly, structural reversibility was also found in a transcranial magnetic stimulation study [29], in which it was suggested that these changes simply reflect vascularization effects. It is widely known that recurrent hypoxia and reoxygenation can induce oxidative stress, increase the production of oxygen free radicals, activate inflammatory pathways and cause vascular endothelial dysfunction [30]. Furthermore, they can induce changes in metabolism. On the other hand, CPAP treatment can reduce the risk of hypoxia, induce revascularization and improve brain metabolism [31]. Thus, CPAP treatment may reinforce brain plasticity that facilitates metabolic recovery in the frontal and connected brain areas.

These findings might also be compatible with previous cognitive function studies, although we did not assess cognitive function in this study. The ACC theory states that the ACC is related to error detection and that part of the circuit is involved in a form of attention that serves to regulate both cognitive and emotional processing [32]. Several placebo-controlled studies conducted on SAS patients have demonstrated the superiority of CPAP over placebo with regard to attention $[33,34]$, which is related to the function of the ACC. The precentral gyrus, as an area of the premotor cortex, plays a role in planning movements, and impaired motor functions in patients with SAS $[14,35,36]$ have been reported to be improved by CPAP $[14,36,37]$.

Unfortunately, the results of this study do not allow us to conclude that brain functional damage caused by hypoxic insults in SAS may be improved by acceptable CPAP treatment. In our patients, hypometabolism in the ACC and precentral gyrus was not fully recovered, and hypometabolism persisted in other regions. Also, Tonon et al. [13] reported that CPAP treatment in SAS subjects had little impact on lowered cortical metabolism measured by magnetic resonance spectroscopy, and they concluded that hypoxic damage in SAS might result in neuronal loss rather than neuronal dysfunction, and this cannot be reversed by CPAP. Some authors have suggested that nocturnal hypoxemia can cause irreversible cerebral damage in SAS. Furthermore, the finding that CPAP can improve deficits related to somnolence, but not those related to hypoxemia, supports this suggestion $[8,14]$. However, hypoxemia could also interfere with the synthesis of neurotransmitters to produce functional revers- 
ible damage. In particular, the cholinergic neurotransmitter system is implicated in many cerebral functions [38] and is sensitive to cerebral hypoxemia of short duration [21]. Therefore, the reduction of hypoxemic events induced by CPAP could normalize the synthesis of neurotransmitters and consequently improve cerebral function. Studies on the impact of long-term CPAP treatment with higher compliance are needed to clarify whether hypoxic cerebral damage in SAS is reversible or irreversible.

This study has several limitations that require consideration. Sham CPAP treatment with subtherapeutic pressure or observation without any treatment could have addressed the placebo effect of CPAP treatment, although it appears unlikely that sham CPAP or observation only could impact on severe SAS with marked hypoxia. In addition, the correction of hypoxia by CPAP was indirectly evaluated using AHI values obtained from the CPAP machine. However, in the clinical setting we routinely estimate the effect of CPAP using AHI values from the CPAP machine, and Mulgrew et al. [39] showed that there were no differences in the AHI between measurements on the CPAP machine and PSG. Measurements of cognitive function could have provided a more comprehensive understanding of hypoxic cerebral damage and its revers- ibility by CPAP in SAS patients. Nonetheless, this is the first study to examine the impact of CPAP on cerebral glucose metabolism using an homogenous group of severe SAS patients who complied with the treatment protocol.

In conclusion, reduced glucose metabolism was observed in severe SAS patients in regions susceptible to hypoxemia, and these abnormalities were found to be modestly corrected by acceptable CPAP treatment. Our results suggest that acceptable CPAP usage cannot completely reverse reduced cerebral glucose metabolism in SAS patients. Further studies are needed to evaluate longterm effects of CPAP treatment with total compliance.

\section{Acknowledgement}

This study was supported by grant 02-2009-028 from the Seoul National University Bundang Hospital Research Fund.

\section{Financial Disclosure and Conflicts of Interest}

The authors have no conflicts of interest to declare in relation to this work.

\section{References}

1 Young T, Peppard PE, Gottlieb DJ: Epidemiology of obstructive sleep apnea: a population health perspective. Am J Respir Crit Care Med 2002;165:1217-1239.

2 Aloia MS, Arnedt JT, Davis JD, Riggs RL, Byrd D: Neuropsychological sequelae of obstructive sleep apnea-hypopnea syndrome: a critical review. J Int Neuropsychol Soc 2004; 10:772-785.

3 McNicholas WT, Bonsigore MR: Sleep apnoea as an independent risk factor for cardiovascular disease: current evidence, basic mechanisms and research priorities. Eur Respir J 2007;29:156-178.

4 Rodenstein D: Sleep apnea: traffic and occupational accidents - individual risks, socioeconomic and legal implications. Respiration 2009;78:241-248.

5 Chung S, Yoon IY, Lee CH, Kim JW: The association of nocturnal hypoxemia with arterial stiffness and endothelial dysfunction in male patients with obstructive sleep apnea syndrome. Respiration 2010;79:363-369.
-6 Morrell MJ, Jackson ML, Twigg GL, Ghiassi R, McRobbie DW, Quest RA, Pardoe H, Pell GS, Abbott DF, Rochford PD, Jackson GD, Pierce RJ, O’Donoghue FJ, Corfield DR: Changes in brain morphology in patients with obstructive sleep apnoea. Thorax 2010; 65:908-914.

7 Torelli F, Moscufo N, Garreffa G, Placidi F, Romigi A, Zannino S, Bozzali M, Fasano F, Giulietti G, Djonlagic I, Malhotra A, Marciani MG, Guttmann CR: Cognitive profile and brain morphological changes in obstructive sleep apnea. Neuroimage 2011;54: 787-793.

8 Pietrini P, Dani A, Raphaelson M, Furey ML, Alexander G, Levine B, Guazzelli M, Rapoport S, Shapiro MC: Cerebral glucose metabolic and neuropsychological dysfunction in patients with untreated sleep apnea syndrome (SAS). Sleep 1998;21:82.

9 Dani A, Pietrini P, Furey M, Freo U, Raphaelson M, Alexander GE, Horwitz B, Guazzelli M, Gemignani A, Shapiro MC: Patients with sleep apnea syndrome (SAS) show neuropsychological impairment and regional cerebral glucose metabolic deficits: a pretreatment positron emission tomography (PET) study. J Sleep Res 1996;5:43.
10 Yaouhi K, Bertran F, Clochon P, Mezenge F, Denise P, Foret J, Eustache F, Desgranges B: A combined neuropsychological and brain imaging study of obstructive sleep apnea. J Sleep Res 2009;18:36-48.

11 Patel SR, White DP, Malhotra A, Stanchina ML, Ayas NT: Continuous positive airway pressure therapy for treating sleepiness in a diverse population with obstructive sleep apnea: results of a meta-analysis. Arch Intern Med 2003;163:565-571.

12 Chung S, Yoon IY, Lee CH, Kim JW: The effects of nasal continuous positive airway pressure on vascular functions and serum cardiovascular risk factors in obstructive sleep apnea syndrome. Sleep Breath 2011;15: 71-76.

13 Tonon C, Vetrugno R, Lodi R, Gallassi R, Provini F, Iotti S, Plazzi G, Montagna P, Lugaresi E, Barbiroli B: Proton magnetic resonance spectroscopy study of brain metabolism in obstructive sleep apnoea syndrome before and after continuous positive airway pressure treatment. Sleep 2007;30:305-311. 
14 Ferini-Strambi L, Baietto C, Di Gioia MR, Castaldi P, Castronovo C, Zucconi M, Cappa SF: Cognitive dysfunction in patients with obstructive sleep apnea (OSA): Partial reversibility after continuous positive airway pressure (CPAP). Brain Res Bull 2003;61:8792.

15 Rechtschaffen A, Kales A: A Manual of Standardized Terminology, Technique, and Scoring System for Sleep Stages of Human Subjects. Los Angeles, Brain Information Service/Brain Research Institute, UCLA, 1968.

16 Sleep-related breathing disorders in adults: recommendations for syndrome definition and measurement techniques in clinical research. The Report of an American Academy of Sleep Medicine Task Force. Sleep 1999;22: 667-689.

17 Iber C, Ancoli-Israel S, Chesson A, Quan S: The AASM Manual for the Scoring of Sleep and Associated Events: Rules, Terminology and Technical Specifications, ed 2. Westchester, American Academy of Sleep Medicine, 2007.

18 Kribbs NB, Pack AI, Kline LR, Smith PL, Schwartz AR, Schubert NM, Redline S, Henry JN, Getsy JE, Dinges DF: Objective measurement of patterns of nasal CPAP use by patients with obstructive sleep apnea. Am Rev Respir Dis 1993;147:887-895.

-19 Lau EY, Eskes GA, Morrison DL, Rajda M, Spurr KF: Executive function in patients with obstructive sleep apnea treated with continuous positive airway pressure. J Int Neuropsychol Soc 2010;16:1077-1088.

20 Castronovo V, Canessa N, Strambi LF, Aloia MS, Consonni M, Marelli S, Iadanza A, Bruschi A, Falini A, Cappa SF: Brain activation changes before and after PAP treatment in obstructive sleep apnea. Sleep 2009;32: 1161-1172.

21 Row BW: Intermittent hypoxia and cognitive function: implications from chronic animal models. Adv Exp Med Biol 2007;618:51-67.
22 Gozal D, Daniel JM, Dohanich GP: Behavioral and anatomical correlates of chronic episodic hypoxia during sleep in the rat. J Neurosci 2001;21:2442-2450.

23 Collop N: Sleep and sleep disorders in chronic obstructive pulmonary disease. Respiration 2010;80:78-86.

24 Shim TS, Lee JH, Kim SY, Lim TH, Kim SJ, Kim DS, Kim WD: Cerebral metabolic abnormalities in COPD patients detected by localized proton magnetic resonance spectroscopy. Chest 2001;120:1506-1513.

25 Canessa N, Castronovo V, Cappa SF, Aloia MS, Marelli S, Falini A, Alemanno F, FeriniStrambi L: Obstructive sleep apnea: brain structural changes and neurocognitive function before and after treatment. Am J Respir Crit Care Med 2011;183:1419-1426.

26 Zimmerman ME, Aloia MS: A review of neuroimaging in obstructive sleep apnea. J Clin Sleep Med 2006;2:461-471.

27 Kempermann G, Gast D, Gage FH: Neuroplasticity in old age: sustained fivefold induction of hippocampal neurogenesis by long-term environmental enrichment. Ann Neurol 2002;52:135-143.

28 Takeuchi H, Taki Y, Sassa Y, Hashizume H, Sekiguchi A, Fukushima A, Kawashima R: Working memory training using mental calculation impacts regional gray matter of the frontal and parietal regions. PLoS One 2011; 6:e23175.

29 May A, Hajak G, Ganssbauer S, Steffens T, Langguth B, Kleinjung T, Eichhammer P: Structural brain alterations following 5 days of intervention: dynamic aspects of neuroplasticity. Cereb Cortex 2007;17:205-210.

30 Logan AG, Bradley TD: Sleep apnea and cardiovascular disease. Curr Hypertens Rep 2010;12:182-188.

31 Buchner NJ, Quack I, Woznowski M, Stahle C, Wenzel U, Rump LC: Microvascular endothelial dysfunction in obstructive sleep apnea is caused by oxidative stress and improved by continuous positive airway pressure therapy. Respiration 2011;82:409-417.

- 32 Bush G, Luu P, Posner MI: Cognitive and emotional influences in anterior cingulate cortex. Trends Cogn Sci 2000;4:215-222.
33 Lim W, Bardwell WA, Loredo JS, Kim EJ, Ancoli-Israel S, Morgan EE, Heaton RK, Dimsdale JE: Neuropsychological effects of 2 -week continuous positive airway pressure treatment and supplemental oxygen in patients with obstructive sleep apnea: a randomized placebo-controlled study. J Clin Sleep Med 2007;3:380-386.

34 Barnes M, McEvoy RD, Banks S, Tarquinio N, Murray CG, Vowles N, Pierce RJ: Efficacy of positive airway pressure and oral appliance in mild to moderate obstructive sleep apnea. Am J Respir Crit Care Med 2004;170: 656-664.

35 Quan SF, Wright R, Baldwin CM, Kaemingk KL, Goodwin JL, Kuo TF, Kaszniak A, Boland LL, Caccappolo E, Bootzin RR: Obstructive sleep apnea-hypopnea and neurocognitive functioning in the Sleep Heart Health Study. Sleep Med 2006;7:498-507.

36 Decary A, Rouleau I, Montplaisir J: Cognitive deficits associated with sleep apnea syndrome: a proposed neuropsychological test battery. Sleep 2000;23:369-381.

37 Engleman HM, Kingshott RN, Wraith PK, Mackay TW, Deary IJ, Douglas NJ: Randomized placebo-controlled crossover trial of continuous positive airway pressure for mild sleep apnea/hypopnea syndrome. Am J Respir Crit Care Med 1999;159:461-467.

38 Furey ML, Pietrini P, Alexander GE, Schapiro $\mathrm{MB}$, Horwitz B: Cholinergic enhancement improves performance on working memory by modulating the functional activity in distinct brain regions: a positron emission tomography regional cerebral blood flow study in healthy humans. Brain Res Bull 2000;51:213-218.

-39 Mulgrew AT, Fox N, Ayas NT, Ryan CF: Diagnosis and initial management of obstructive sleep apnea without polysomnography: a randomized validation study. Ann Intern Med 2007;146:157-166. 